\title{
Óbitos Por Violência Retratados Pela Mídia Televisiva no Sudoeste de Mato Grosso
}

\author{
Vagner Ferreira do Nascimento, ${ }^{1}$ Thalise Yuri Hattori, ${ }^{2}$ \\ Ana Cláudia Pereira Terças, ${ }^{3}$ Ana Carla Ferreira Picalho ${ }^{4}$
}

\begin{abstract}
RESUMO
Objetivo: Caracterizar os óbitos por violência retratados pela mídia televisiva no Sudoeste de Mato Grosso, Brasil. Método: Estudo exploratório, com abordagem quantitativa, utilizando dados de acesso e domínio público a partir de um telejornal, em canal aberto, sediado na mesorregião do Sudoeste do Mato Grosso, Brasil. A coleta de dados foi realizada entre junho e outubro de 2015, por meio de gravação diária dos programas. Resultados: Os óbitos por violência evidenciados nesse estudo foram majoritariamente de pessoas do gênero masculino, jovens, sendo causados em sua maioria por ferimento de arma branca seguidos de arma de fogo, ocorrendo normalmente nos finais de semana e no período noturno. Conclusão: Esse quadro acarreta diretamente impacto negativo na sociedade, principalmente na força produtiva do país, na desestruturação familiar, no aumento de desconfiança nas relações interpessoais ou no fomento de comportamentos hostis diante de conflitos comuns do cotidiano.
\end{abstract}

Palavras-chave: Saúde pública. Violência. Causas externas. Morte.

DEATHS FROM VIOLENCE PORTRAYED BY THE TELEVISION MEDIA IN THE SOUTHWEST OF MATO GROSSO

\section{ABSTRACT}

Objective: To characterize the violence deaths portrayed by the television media in Southwest Mato Grosso, Brazil. Method: Exploratory study, using a quantitative approach, using access data and public domain from a television news channel, open channel, based in the mesoregion of the Southwest of Mato Grosso, Brazil. Data collection was performed between June and October 2015, through daily recording of programs. Results: Violence deaths evidenced in this study were mostly of young men, mostly caused by gunshot wounds followed by firearms, usually occurring on weekends and at night. Conclusion: This situation directly implies a negative impact on society, especially on the country's productive strength, on family disorganization, on increasing mistrust in interpersonal relations or on fomenting hostile behavior in the face of everyday conflicts.

Keyworks: Public health. Violence. External causes. Death.

Recebido em: 4/12/2016

Alterações requeridas em: 12/2/2019

Aceito em: 9/5/2019

\footnotetext{
Docente-adjunto da Universidade do Estado de Mato Grosso (Departamento de Enfermagem - Unemat), campus universitário de Tangará da Serra. Participa dos grupos de pesquisa CNPq: NPEPS (Unemat); Cultura, Política e Sociedade (Unemat) e Relações de Gênero, Violências e Comunicação (Unemat). Conselheiro Coren MT. vagnerschon@hotmail.com

${ }^{2}$ Docente-assistente da Universidade do Estado de Mato Grosso (Departamento de Enfermagem - Unemat), campus universitário de Tangará da Serra. thalisehattori@gmail.com

${ }^{3}$ Docente-adjunta da Universidade do Estado de Mato Grosso (Departamento de Enfermagem - Unemat), campus universitário de Tangará da Serra. Docente permanente do Programa de Pós-Graduação em Saúde Coletiva da Universidade Federal de Mato Grosso (ISC/UFMT). enfanacnp@gmail.com

${ }^{4}$ Enfermeira. Graduada em Enfermagem pela Universidade do Estado de Mato Grosso (Unemat), campus universitário de Tangará da Serra. annnakkarlla@hotmail.com
} 


\section{INTRODUÇÃO}

A Organização Mundial da Saúde (OMS) estima que ocorreram 468 mil assassinatos no mundo em 2015 e de acordo com a agência da Organização das Nações Unidas (ONU), outras 152 mil pessoas foram mortas em guerras e confrontos. No Brasil, as taxas de mortalidade estão associadas principalmente a causas externas. Além disso, na América Latina, o país só é menos violento que a Colômbia, Venezuela, El Salvador e Honduras (BRASIL, 2015).

Essas mortes são provocadas principalmente por violências que compõem um quadro dramático e comum na sociedade, consequentemente representam uma questão importante para as políticas públicas (YWATA et al., 2008) e para a economia do país, uma vez que a violência e outras experiências traumáticas, além de extinguirem forças de trabalho e desacelerarem a produtividade pela precocidade de óbitos, podem fortalecer condições de conflitos e pobreza (MOYA, 2018).

Vários fatores contribuem para a ocorrência e aumento de óbitos por violência. Na capital do Estado de Mato Grosso, entre esses fatores destacam-se a maternidade na adolescência, sobrecarga de papéis maternos, consumo de álcool e drogas, conflitos familiares e episódios de morte anterior por violência na família (MARTINS; JORGE, 2013). Diante dessas e de outras desigualdades que contribuem para o aumento dos óbitos por violência, há necessidade de direcionar permanentemente o olhar para esse fenômeno, promovendo maior celeridade na incorporação dessa pauta nas agendas de gestores e nos diálogos gerados a partir dessa problemática social (GUZZO et al., 2014).

Esse movimento para enfrentamento da violência pode ser alavancado com apoio de dispositivos e instrumentos sociais que possuem acesso popular e proximidade comunitária. Para tanto alguns desses meios, como a mídia, reproduzem a cultura da violência como uma resposta ao cotidiano social que busca combater, proteger ou livrar o cidadão do perigo (CARVALHO; FREIRE; VILAR, 2012).

Por outro lado, a violência na perspectiva da mídia também é capaz de criar ou manter significados. As imperfeições dos indivíduos, conhecidas como contrárias à virtude como luxo, lascívia, avareza e orguIho, são reforçadas pelo destaque ao sofrimento das vítimas. A realidade e o sofrimento do outro, comparados à indignação, à compaixão, aos sentimentos de perda e à angústia, intensificam as virtudes do cida- dão comum, que pode se autojulgar como justo, solidário ou generoso, conforme a situação lhe permitir (CARVALHO; FREIRE; VILAR, 2012).

O papel pensado e exercido pela mídia há mais de 20 anos revela a importância da divulgação e reflexão de diversas visões sobre a violência praticada na sociedade. Esse papel vai muito além da cobertura das notícias sobre violência, ela mobiliza as pessoas a pensarem e agirem diante desses fatos noticiados. Cabe ressaltar ainda que apesar da magnitude e importância do tema há escassez de trabalhos que abordem os óbitos por violência retratados pela mídia televisiva, o que amplia a necessidade de um investimento maior sob a égide dos meios de comunicação e informações que consigam compreender de forma mais aprofundada as diferentes formas de apresentação desse fenômeno. Assim sendo, este estudo objetivou caracterizar os óbitos por violência retratados pela mídia televisiva no Sudoeste de Mato Grosso, Brasil.

\section{MATERIAIS E MÉTODOS}

Trata-se de um estudo exploratório, com abordagem quantitativa, utilizando dados compartilhados, a partir de um telejornal de canal aberto, sediado na mesorregião do Sudoeste do Mato Grosso, Brasil. A escolha dessa localidade para o estudo deu-se pela identificação do movimento conhecido como interiorização da violência, o qual evidencia o deslocamento da violência letal dos municípios de grande porte para os de médio porte (CARNEIRO; FRAGA, 2012).

Essa região é formada pela união de 22 municípios agrupados em três microrregiões, com área total de 72.064,499 km² e com 306.183 habitantes. Apresenta características importantes para o fenômeno da violência, como baixo nível de desenvolvimento social e econômico (IBGE, 2014).

Como critério de inclusão foi estabelecido: emissora em funcionamento há mais de 72 meses, com transmissões de telejornais do meio-dia. Excluíram-se as emissoras com alcance de cobertura inferior a cinco municípios. Assim, das sete emissoras que poderiam ser incluídas no estudo, atendeu aos critérios somente uma delas. A definição desses critérios baseou-se no ponto de vista de que emissoras com maior tempo de criação, entendidas como consolidadas e com maior abrangência geográfica, poderiam disseminar informações sobre a realidade para um número maior de pessoas.

A coleta de dados foi realizada no período de junho a outubro de 2015, por meio de gravação diária dos programas em DVD, com duração média de $60 \mathrm{mi}$ - 
nutos. Participaram da equipe de trabalho quatro pesquisadores. A escolha desse recorte temporal ocorreu por contemplar nesse período o calendário de grandes festividades e eventos culturais da região, o que poderia gerar mais casos de violência.

Para análise dos dados iniciou-se com a visualização de 79 telejornais gravados, selecionando aqueles que possuíam reportagens com ocorrências de óbitos por violência. Neste estudo considerou-se óbitos por violência os homicídios, suicídios e acidentes de trânsito/transporte (WAISELFISZ, 2013). Assim, após avaliação dos programas do telejornal selecionado, obteve-se o quantitativo de oito óbitos por violência.

A partir dessa seleção ocorreu a compilação destes óbitos selecionados em planilhas de Excel, de modo a favorecer a identificação dos elementos "Quem", "Como", "Onde" e "Quando", referindo a ocorrência dos óbitos. Após essa etapa, houve o agrupamento e organização desses elementos em forma descritiva, discutindo os achados com referenciais da literatura científica.

O estudo não necessitou de apreciação e aprovação de Comitê de Ética em Pesquisa, pois utiliza dados disponíveis a acesso público e irrestrito.

\section{RESULTADOS E DISCUSSÃO}

A violência caracteriza-se como a prática contra si mesmo, contra outra pessoa ou contra um grupo que resulte em morte, sofrimento, danos, desenvolvimento ou privação que pode ocorrer em diferentes contextos, como o uso da força física ou de poder com a utilização de ameaças, intimidação ou negligência (OMS, 2014). O aumento das taxas de morbimortalidade por violência exposto pela mídia televisiva no país deixa claro os constantes risco à saúde da população em geral, constituindo, dessa forma, um problema prioritário de saúde pública (DUARTE et al., 2012).

Os homens são os mais atingidos pela diversidade de formas de violência no Brasil (ANDRADE-BARBOSA et al., 2013). No presente estudo, $89 \%$ dos casos de óbitos por violência retratados na mídia eram sujeitos do gênero masculino. Esse resultado também assemelha-se a outras pesquisas nacionais e internacionais (CAMPOS et al., 2015; COSTA, TRINDADE, SANTOS, 2014; CRAWFORD, 2014; HUMMER et al., 2014; PIOVEZAN et al., 2018; TRINDADE et al., 2015; ZIRABA, KYOBUTUNGI, ZULU, 2011).

O sexo masculino a partir da adolescência está mais suscetível à violência extrafamiliar devido às questões sociais e culturais e maior exposição a situações de risco (COUTINHO et al., 2013; OLIVEIRA, 2017;
PIOVEZAN et al., 2018; SILVA; DELL'AGLIO, 2016). Situações como maior independência, prática de condutas violentas nos aspectos diversos relacionados com os veículos, adicionadas à violência e à sexualidade, favorecem certas práticas de risco que levam a prejuízos à saúde e à morte por causas específicas (VIANA et al., 2011).

Além disso, a educação baseada em um modelo masculino estereotipado que apresenta a agressividade, a competividade e a repreensão dos sentimentos de afeto provoca nos homens valores que envolvem um desfecho violento (RODRÍGUEZ et al., 2013). Foi comprovado que indivíduos que residem em regiões desfavorecidas ou em condições precárias têm uma maior chance de se tornarem vítimas de morte violenta, especialmente de homicídio (VIANA et al., 2011). Mundialmente o perfil epidemiológico da mortalidade por homicídios caracteriza-se pelo predomínio de pessoas jovens, do sexo masculino, negras ou descendentes dessa raça/etnia, pertencentes ao estrato socioeconômico menos favorecido e com baixo nível de escolaridade (SOUZA; LIMA; BEZERRA, 2010). Tais resultados condizem com as associações (sexo, faixa etária jovem, experiência de violência anterior, menor escolaridade dos pais, renda e estrutura familiar) encontradas por Martins e Jorge (2013).

No estudo observou-se que $44 \%$ dos óbitos corresponderam à violência envolvendo ferimentos por arma branca (FAB) tipo perfurocortantes, seguidos pelos ferimentos por arma de fogo (FAF), com $22 \%$, e empatados com $11 \%$ estão aqueles causados por atropelamento, perfuração do crânio e tórax. As características epidemiológicas das causas externas fatais dessa região estudada assemelham-se ao estudo realizado em Alagoas (TRINDADE et al., 2015) e em pesquisa efetivada nas Filipinas, em que se reafirma que os mecanismos mais comuns de lesões por violência ocorrem por arma branca (32,9\%), tendo comumente como consequência óbito por hemorragia $(66,7)$ nas primeiras 24 horas (CONSUNJI et al., 2011).

Apesar de as lesões por arma branca ainda serem mais comuns, estudo realizado na Suécia revela crescimento de lesões por arma de fogo, sendo mais letais e sérios quando atingem a cabeça, tórax e abdômen (KHOSHNOOD, 2018). Pesquisa em Minas Gerais reforça esse cenário e ressalta os anos potenciais de vida perdidos (CAMARGO et al., 2018).

Nos homicídios, a arma de fogo está presente na maioria das ocorrências (ALVES et al., 2014), da mesma forma como retratado em estudos anteriores realizados na capital do Estado de Mato Grosso (MARTINS; JORGE, 2013). Nessa mesma vertente, estudo conduzi- 
do em uma região da África aponta que a maior parte dos óbitos por violência ocorrem por lesões intencionais $(51,5 \%)$, normalmente pelas intensas disputas do tráfico de drogas (ZIRABA; KYOBUTUNGI; ZULU, 2011).

Embora as armas de fogo superem em incidência outros agentes nas agressões interpessoais no Brasil, as armas brancas se mantêm como instrumento mais frequentemente utilizado, particularmente em conflitos domiciliares (WAISELFISZ, 2013). Em geral, nos estudos internacionais, a arma de fogo também é a mais utilizada nos casos de homicídios (SOUZA; LIMA; BEZERRA, 2010; SOUZA et al., 2012). Acredita-se que esses resultados possam ser explicados pela simbologia que esse instrumento representa associado com o poder de vida ou morte, além do precoce contato desde a infância na forma de brinquedo, que faz com que o torne comum e aceitável no universo masculino (SOUZA et al., 2012).

A distribuição da faixa etária dos óbitos foi bem distinta, estando entre 16 e 45 anos, mas em alguns casos a mídia não apresentou esses dados, o que impediu melhor caracterização das ocorrências e dos sujeitos envolvidos. Em um estudo nacional, nas principais capitais do Brasil, pessoas de 20 a 29 anos, sobretudo em Recife e no Rio de Janeiro e com 60 ou mais anos de idade no Distrito Federal, Manaus e Curitiba, são as mais afetadas pela mortalidade por causas externas (MINAYO; DESLANDES, 2009). Um estudo realizado na Jamaica confirmou esses resultados, apontando essa mesma faixa etária para os jovens do país (CRAWFORD, 2014).

Verificou-se também que a mortalidade por homicídio no sexo masculino teve um aumento em todas as faixas etárias, com destaque para as idades entre 15-30 anos (CAMARGO et al., 2018; CAMPOS et al., 2015; CORRÊA; SOUZA, 2011; COSTA; TRINDADE; SANTOS, 2014; OLIVEIRA, 2017; TRINDADE et al., 2015). Estudiosos afirmam que as mortes violentas estão como primeira causa de óbito entre os adolescentes e adultos jovens (COSTA; TRINDADE; SANTOS, 2014; WAISELFISZ, 2013, 2011). Entre as razões do acometimento entre os mais jovens encontram-se exclusão da educação, falta de perspectiva de futuro, à pobreza, vulnerabilidade às situações que envolvem maior risco e exclusão social (ZANDOMENIGHI; MARTINS; MOURO, 2011; WAISELFISZ, 2013).

Há ainda uma possível teoria de que a violência exposta pela mídia possa estar relacionada ao aumento da violência entre adultos jovens por interferir nos mecanismos inibitórios do controle cognitivo mediados pelo córtex frontal (HUMMER et al., 2014). Já entre crianças de 8-12 anos comprovou-se que assistir programas que retratam a violência estimula a conduta agressiva dessas crianças, independentemente da localização geográfica, sexo, nível econômico. Acredita-se que a conduta agressiva é fomentada pelo meio televisivo pelo fato de a imitação ser o primeiro mecanismo de aprendizagem da criança, chegando a julgar essa ação agressiva como uma conduta apropriada e posteriormente perpetuando-a na adolescência e vida adulta (FLORES; CARRASCO; VICENTE, 2011).

Outra explicação plausível seria o fato dos jovens, pelas características biológicas e psíquicas peculiares dessa fase da vida, que estão permeadas pelo sentimento de insegurança e conflitos internos, associadas às discrepâncias socioeconômicas e culturais, precipitarem o aumento do comportamento violento e a conduta de risco como forma de exteriorizar suas emoções/sensações. Eles sentem a necessidade de se identificar com um mundo que consiga oferecer suporte emocional para enfrentarem e desfrutarem a vida, o que implicitamente envolve a possibilidade de morte. Assim, abuso de drogas (licitas ou ilícitas), promiscuidade sexual, direção imprudente, comportamentos compulsivos e o imediatismo são formas de reivindicação sobre uma realidade de vida do jovem que tem uma visibilidade para a falta de sentido dos projetos de vida (CORRÊA; SOUZA, 2011).

Quanto ao perfil dos envolvidos no ato violento, a mídia não o retratou em todos os casos, no entanto revelou maior especificidade daqueles sujeitos que possuíam algum envolvimento com drogas, como se correlacionassem pelo discurso jornalístico o óbito ou a circunstância deste a essa prática. Outro estudo que analisou os principais meios de comunicação escrita no Brasil sobre a saúde relacionada ao uso de drogas também relacionou o seu consumo com casos de dependência ou situações de violências acrescida de um crescente consumo (NOTO et al., 2003).

Os óbitos por violência foram bem distribuídos por todo o território do sudoeste mato-grossense, ou seja, várias cidades foram pontos de violência e nessas a zona urbana foi o local de ocorrência mais comum. Outro estudo confirma essa concentração de casos, relatando que as aglomerações urbanas são vistas como um fator predisponente ou facilitador para a ocorrência desse fenômeno, destacando-se que a urbanização não planejada, desigualdades socioeconômicas e a pobreza também são fatores relevantes (COSTA; TRINDADE; SANTOS, 2014).

O local da morte por violência retratada pela mídia televisiva deu-se em sua maioria no local em que ocorreu a violência, em espaço urbano aberto. Resultados semelhantes foram encontrados por Trin- 
dade et al. (2015), referindo que os principais locais das ocorrências foram as vias públicas $(75,1 \%)$. A ocorrência dos homicídios no local onde a vítima foi agredida pode indicar a intencionalidade da agressão, não Ihe permitindo possibilidade de sobrevivência (COSTA; TRINDADE; SANTOS, 2014; TRINDADE et al., 2015).

Quanto à distribuição dos óbitos, verificou-se que grande parte das mortes ocorreu durante os meses de julho e outubro e uma pequena porcentagem dos óbitos ocorreu no mês de agosto. Nos demais meses, durante a pesquisa, a mídia não relatou nenhuma morte por violência. Esses meses em que não foram identificadas nenhuma morte por violência devem ser investigados a fim de constatar se o padrão temporal dos óbitos dessa região ocorrem por aspectos culturais (períodos festivos) ou associado a outros fatores. Segundo Matos e Martins (2009), quanto à sazonalidade em Cuiabá, não há um mês específico em que se sobressaiam as fatalidades decorrentes da violência, visto que em todos os meses estes casos estiveram presentes em grande número.

Na distribuição temporal, o final de semana foi o mais representativo quanto à ocorrência de óbitos por violência, prevalecendo o domingo com a maior proporção dos casos. Observou-se que grande parte dos óbitos ocorreu no período noturno, seguido pelo período vespertino. Esses dados corroboram com outros estudos nos quais as violências ocorreram, em sua maioria, nos finais de semana e após o entardecer (COSTA; TRINDADE; SANTOS, 2014; FREITAS et al., 2017; TRINDADE et al., 2015). Acredita-se que a circulação dos indivíduos em horários não usuais em locais públicos associada com o consumo excessivo de bebidas alcoólicas (FREITAS et al., 2017; PIOVEZAN et al., 2018) e outras drogas podem contribuir para o envolvimento e/ou exposição a situações de violência que podem justificar os resultados encontrados (TRINDADE et al., 2015).

A violência é um problema que afeta diretamente a saúde, trazendo sequelas à família da vítima e à comunidade. Em razão disso, novos mecanismos sociais e políticos, bem como novas posturas individuais e coletivas diante da violência devem ser estabelecidos, a fim de minimizar ou desacelerar perdas e prejuízos para a humanidade e outras gerações. Para tanto, há necessidade de que outras pesquisas desse caráter tenham continuidade, a fim de construir conhecimentos sólidos sobre as mortes violentas, as relações de poder e as representações sociais desse fenômeno, que ainda se apresenta como um grande desafio para a sociedade (SOUZA; SOUZA; PINTO, 2014).

\section{CONCLUSÃO}

Percebe-se que o fenômeno da violência tem ocupado lugar de destaque e preocupação na sociedade, principalmente nos meios de comunicação. Após o término deste estudo, porém, verificou-se que a mídia investigada não apresenta os casos de violência de forma completa para a compreensão da problemática, deixando de veicular algumas informações importantes para a caracterização das ocorrências, como cenário e perfil dos envolvidos.

Os óbitos por violência evidenciados neste estudo concentraram-se no gênero masculino, em adultos jovens, por ferimento de arma branca, seguidos de arma de fogo, ocorridos normalmente nos finais de semana e no período noturno. Esse quadro implica diretamente um impacto negativo na sociedade, principalmente na força produtiva do país, na desestruturação familiar, no aumento de desconfiança nas relações interpessoais ou no fomento de comportamentos hostis diante de conflitos comuns do cotidiano.

Dada essa importância, o monitoramento dos óbitos por violência mostra-se fundamental ao objetivar possibilidades de intervenção, tanto no âmbito preventivo como no processo de cuidado aos sujeitos e famílias pós-violência. Assim, o incentivo à criação de centros de referências estaduais e municipais, como os Núcleos de Prevenção às Violências e Promoção da Saúde (NPVPS) pode ser uma estratégia favorável quando articulados com a rede de atenção à saúde e de atendimento de assistência social, na medida em que a finalidade não seja apenas notificar e publicar os casos, mas comprometer-se com a cultura de paz e contextos de vida mais saudáveis.

A partir disso, sugere-se novas investigações, principalmente por meio de estudos longitudinais com monitoramento da população, de tal forma que a compreensão desse fenômeno possa ser ampliada.

\section{REFERÊNCIAS}

ALVES, W. A. et al. Violência letal em Maceió-AL: estudo descritivo sobre homicídios, 2007-2012. Epidemiol. Serv. Saúde, v. 23, n. 4, p. 731-740, 2014.

ANDRADE-BARBOSA, T. L. et al. Mortalidade masculina por causas externas em Minas Gerais, Brasil. Ciênc. Saúde Coletiva, v. 18, n. 3,13; p. 711-719, 2013.

BRASIL. Impacto da violência na saúde dos brasileiros. Brasília: Ministério da Saúde, 2015.

CAMARGO, F. C. et al. Evolução temporal dos anos potenciais de vida perdidos em óbitos por agressão. Rev. Enferm. Atenção Saúde, v. 7, n. 2. p. 68-82, 2018.

CAMPOS, M. R. et al. Morbidity and mortality associated with injuries: results of the Global Burden of Disease study in Brazil, 2008. Cad Saúde Pública, v. 31, n. 1, p. 121-136, 2015. 
CARneiro, A. A.; FRAgA, C. K. A Lei Maria da Penha e a proteção legal à mulher vítima em São Borja no Rio Grande do Sul: da violência denunciada à violência silenciada. Serv. Soc. Soc., v. 110, p. 369-397, 2012.

CARVALHO, D. W.; FREIRE, M. T.; VILAR, G. Mídia e violência: um olhar sobre o Brasil. Rev. Panam. Salud Publica, v. 31, n. 5, p. 435-8, 2012.

CONSUNJ, R. J. et al. A profile of deaths among trauma patients in a university hospital: The Philippine experience. $J$. Inj. Violence Res., v. 3, n. 2, p. 85-89, 2011.

CORRÊA, C. S.; SOUZA, S. J. Violência e vulnerabilidades: os jovens e as notícias de jornal. Fractal: Revista de Psicologia, v. 23, n. 3, p. 461-486, 2011.

COSTA, F. A. M. M.; TRINDADE, R. F. C.; SANTOS, C. B. Deaths from homicides: a historical series. Rev. Latinoam. Enferm, v. 22, n. 6, p. 1.017-1.025, 2014.

COUTINHO, R. X. et al. Prevalência de comportamentos de risco em adolescentes. Cad. Saúde Coletiva, v. 21, n. 4, p. 441-449, 2013.

CRAWFORD, T. V. et al. Epidemiological Features of violence-related Injuries in Jamaica. J. Clin. Diagn. Res, v. 8, n. 11, p. JC01-JCO4, 2014.

DUARTE, E. C. et al. Associação ecológica entre características dos municípios e o risco de homicídios em homens adultos de 20-39 anos de idade no Brasil, 1999-2010. Ciênc. Saúde Coletiva, v. 17, n. 9, p. 2.259-2.268, 2012.

FLORES, V. C.; CARRASCO, G. C.; VICENTE, S. L. Contenidos televisivos violentos asociados a la conducta agresiva de niños de 8 a 12 años. Rev. Enferm. Herediana, v. 5, n. 2, p. 49-55, 2011.

FREITAS, R. A. G. et al. Perfil dos casos de violência socorridos por um Serviço de Atendimento Móvel de Urgência Estadual. Rev. Bras. Pesq. Saúde, v. 19, n. 2, p. 6-14, 2017.

GUZZO, P. C. Práticas de saúde aos usuários em situação de violência: da invisibilidade ao (des)cuidado integral. Rev. Gaúcha Enferm., v. 35, n. 2, p. 100-1.105, 2014.

HUMMER, T. A. et al. Association of television violence exposure with executive functioning and white matter volume in young adults males. Brain Cogn, v. 14, n. 88, p. 26-34, 2014.

IBGE. Instituto Brasileiro de Geografia e Estatística. Censo Demográfico 2014: Resultados do universo. Disponível em: http://cidades.ibge.gov.br/xtras/perfil.php?codmun=510795. Acesso: em 5 fev. 2015.

KHOSHNOOD, A. Firearm-related violence in Sweden - A systematic review. Aggression and Violent Behavior, v. 42, p. 43-51, 2018.

MARTINS, C. B. G.; JORGE, M. H. P. M. Óbitos por causas externas em Cuiabá, 0 a 24 anos: perfil das vítimas e famílias segundo a intencionalidade. Rev. Bras. Epidemiol, v. 16, n. 2, p. 454-468, 2013.

MATOS, K. F.; MARTINS, C. B. G. Perfil epidemiológico da mortalidade por causas externas em crianças, adolescentes e jovens na capital do Estado de Mato Grosso, Brasil, 2009. Epidemiol. Serv. Saúde, v. 21, n. 1, p. 43-53, 2012.

MINAYO M. C. S.; DESLANDES S. F. Análise da implantação da rede de atenção às vítimas de acidentes e violências segundo as diretrizes da Política Nacional de Redução da Morbimortalidade sobre Violência e Saúde. Ciênc. Saúde Coleti$v a$, v. 14, n. 5, p. 1.641-1.649, 2009.
MOYA, A. Violence, psychological trauma, and risk attitudes: Evidence from victims of violence in Colombia. J. Dev. Econ., v. 131, p. 15-27, 2018.

NOTO, A. R. et al. Drogas e saúde na imprensa brasileira: uma análise de artigos publicados em jornais e revistas. Cad. Saúde Pública, v. 19, n. 1, p. 69-79, 2003.

OLIVEIRA, J. V. Retratos da violência urbana e da criminalidade em Boa Vista - Roraima: a capital mais setentrional do Brasil. Rev. Cad. Campo, n. 22, p. 245-270, 2017.

OMS. Strengthening the role of the health system in addressing violence, in particular against women and girls, and against children. Sixty Seventh World Health Assembly Resolution, 2014. Available from: http://apps.who.int/gb/ebwha/ pdf_files/WHA67/A67_R15-en.pdf?ua=1. Cited: 5 Feb. 2015. PIOVEZAN, L. N. C. et al. Análise das fichas de notificação de violência emitidas por serviços de saúde da região de Barbacena. Rev. Med. Minas Gerais, v. 28, Supl 5, e-S280502, 2018.

RODRÍGUEZ, M. V. et al. Mortalidad por causas violentas en hombres de la provincia de Santiago de Cuba durante 2011. Medisan, v. 17, n. 5, p. 767-773, 2013.

SILVA, D. G.; DELL'AGLIO, D. D. Exposure to Domestic and Community Violence and Subjective Well-Being in Adolescents. Paidéia, Ribeirão Preto, v. 26, n. 65, p. 299-305, 2016. SOUZA, E. R. et al. Estudo multicêntrico da mortalidade por homicídios em países da América Latina. Ciênc. Saúde Coletiva, v. 17, n. 12, p. 3.183-3.193, 2012.

SOUZA, E. R.; LIMA, M. L. C.; BEZERRA, E. A. D. Homicides in Brazil: Evolution and Impacts. In: LOVISI, G. M.; MARI, J. J.; VALENCIA, E. S. The Psycological Impact of Living under violence and poverty in Brazil. Nova York: Nova Science Publishers Inc, 2010.

SOUZA, T. O.; SOUZA, E. R.; PINTO, L. W. Evolução da mortalidade por homicídio no Estado da Bahia, Brasil, no período de 1996 a 2010. Ciênc. saúde coletiva. 2014, v. 19, n. 6, p. 1.889-1.900.

TRINDADE, R. F. C. et al. Map of homicides by firearms: profile of the victims and the assaults. Rev. Esc. Enferm. USP, 49(5), 748-75, 2015.

VIANA, L. A. C. et al. Social inequalities and the rise in violent deaths in Salvador, Bahia State, Brazil: 2000-2006. Cad. Saúde Pública, n. 27, Suppl. 2, p. s298-s308, 2011.

WAISELFISZ, J. J. Mapa da violência 2011: os jovens no Brasil. São Paulo: Instituto Sangari, 2011. Disponível em: http://www.sangari.com/mapadaviolencia/pdf2011/MapaViolencia2011.pdf. Acesso em: 2 out. 2014.

WAISELFISZ, J. J. Mapa da violência 2013: mortes matadas por armas de fogo. Rio de Janeiro: Cebela; Flacso, 2013. Disponível em: http://www.mapadaviolencia.org.br/pdf2013/ MapaViolencia2013_armas.pdf. Acesso em: 2 out. 2014.

YWATA, A. X. C. et al. Custos das mortes por causas externas no Brasil. Rev. Bras. Biom, v. 26, n. 3, p. 23-47, 2008.

ZANDOMENIGHI, R. C.; MARTINS, E. A. P.; MOURO, D. L. Ferimento por projétil de arma de fogo: um problema de saúde pública. Rev. Min. Enferm, v. 15, n. 3, p. 412-20, 2011. ZIRABA, A. K.; KYOBUTUNGI, C.; ZULU, E. M. Fatal Injuries in the Slums of Nairobi and their Risk Factors: Results from a Matched Case-Control Study. Journal of Urban Health: Bulletin of the New York Academy of Medicine, v. 88, Suppl. 2, p. 256-265, 2011. 